\title{
Study on Phonetics Acquisition of English Learners from Regions of Prominent Dialects in Anhui Province from the Perspective of Optimality Theory*
}

\author{
Huazhen Wang \\ School of Foreign Languages \\ Anhui Sanlian University \\ Hefei, China 230601
}

\begin{abstract}
Based on Optimality theory and the existing research result at home and abroad, this paper discover the influence of Anhui dialect on undergraduates' English phonetic learning through the long-term phonetics teaching and experimental teaching, probes into the problems in phonetic transfer of graduates in typical dialectal areas of Anhui province and its reasons, and formulate new corrective teaching strategy. Through long-term teaching experiment and practical investigation, the author explores and analyzes the phonetic phenomenon of negative transfer from Anhui dialects and the distribution area. Then feasible phonetic correction approach is given according to the phonetic feature of undergraduates.
\end{abstract}

Keywords-Optimality theory; Anhui dialects; English Phonetics; correcting strategies

\section{INTRODUCTION}

Optimality theory is a kind of generative phonology theory put forward by Bruce Tesa and Paul Smolensky in the early 1990s. The domestic research in this field is still in initial stage, so there are not many relevant research papers that can be searched and most of them are introduction and theoretical elucidation, without underlying applied researches. This study starts with the study of the rankings and moving paths of English learners in dialectal areas during each acquisition stages to explore the internal mechanisms that dominate the phonological acquisition of English learners under OT frame. With the help of $\mathrm{P}$ raat, a phonetic software in experimental phonetics, the phonetic system of English and Chinese is analyzed and marked and empirical research is made on the tactics of accent in English words, phrases and sentences, which are expressed in the order ranking form of constraints to approach to native English speakers.

\section{THE RESEARCH STATUS AT HOME AND ABROAD}

In the field of foreign language studies, Optimality Theory is one of the most important topics studied by many linguists at home and abroad. It is the development of The

*Fund Project: Humanities and Social Sciences Key Project of Education Department of Anhui Province (Project No. SK2015A638); Quality Project of Anhui Sanlian College (Project Number: 14zlgc025)
Sound Pattern of English (SPE in short, Chomsky \& Halle, 1968). Once born, it has far-reaching influence on the entire linguistic field and has been widely used in children's language acquisition, second language acquisition, syntax and semantics as a linguistic theory. The first scholar in China to introduce Optimality theory is Wang Jialing (1995). His article entitled "Optimality Theory" published in "Foreign Linguistics" first introduced the basic content of this theory and opened the prelude to the study of Optimality theory in China. Later, some scholars (Zhong Rongfu, 1995; Li Bing, 1998; Zuo Yan, 1999; Gong Qi, 2000; Ma Qiuwu, 2001; Wang Fufang, 2003) have written articles to introduce the theoretical framework of Optimality theory and its latest progress. The introduction of theory involves all aspects of Optimality theory. The core concept of Optimality theory is the constraints and hierarchical ranking of constraints. The general constraints fall into two categories: the marked constraints and faithful constraints. Faithful constraints require that the output be consistent with the input. This study comparatively analyze the English and Chinese phonetic system under the OT frame, combining with the actual survey of undergraduates' pronunciation in Anhui dialect area to study the phenomenon of undergraduates phonetic transfer and construct a feasible long-term research mechanism and phonetic teaching reform project for correcting phonetics strategies.

\section{ENGLISH PRONUNCIATION AND ANHUI DIALECT}

Language is composed of pronunciation, and its role is communication, of which the main form is oral communication that cannot be separated from the pronunciation of language. English pronunciation cannot go beyond the scope that segment and phoneme allow. Otherwise it will lead to deviation of pronunciation. Anhui province stretches across Jianghuai area, with complex dialect distribution, which can be divided into the Mandarin dialect of Central Plains in northern Anhui, Mandarin of Jianghuai in central Anhui, Gan dialect in Western Anhui, $\mathrm{Wu}$ dialect of Xuanzhou in southern Anhui, Hui dialect in Southern Anhui and Guest Language in Southern Anhui. Its negative transfer impact on English phonetic learning is reflected in the segments and phonemes. 


\section{A. Optimization Study on the Transfer Phenomenon of Anhui Dialect to English Phonetic}

Anhui dialect refers to the dialect used within Anhui Province. Anhui covers the three major basins of Huaihe River, the Yangtze River, and Xin'an River, with great differences in culture between north and south. Dialect pronunciation refers that under the influence of the Chinese dialect pronunciation system, some people in the dialectal areas retain the pronunciation of consonants, vowels and tones of the dialect when using mandarin. Domestic scholars also make explanatory research on the Tone Sandhi of dialect introduced by some scholars, mainly including "Optimality analysis of tonal sandhi in Beijing dialect" (Wang Jialing, Xinjiang University Language and Culture International Symposium Proceedings, 2002), "Optimality theory and tonal sandhi and light tone in Tianjin dialect" (Wang Jialing, Studies of The Chinese Language, 2002), "Optimality Analysis of tonal sandhi and neutral tones in trisyllabic sequence in Hefei Dialect" (Kong Huifang, Journal of Anhui Agricultural University, 2004) , "restudy the mystery of tonal sandhi in Tiandi dialect" (Ma Qiuwu, Contemporary Linguistics, 2005) and so on. Another example is the Optimality analysis of tone in Hefei dialect, which firstly uses the experimental phonetic method, and use the voice analysis software to make comprehensive voice description of Hefei dialect tone. Then analysis is made on the tone sandhi and light tone of two-syllabic words in Hefei dialects in the framework of Optimality theory.

\section{B. Collecting the Survey Data through Audio Recording Interview}

The recording software CoolEditpro.2.0 is adopted to let the students to read aloud the selected 100 words, and classify the sound corpus according to the classification standard of phonological knowledge. The categories are to be taken as shown in "Fig. 1":

- A. accent
a. accent of simple word
b. accent of compound word

- B. weak pronunciation

- C. phoneme

- D. coherent speech

a. rhythm

Assimilation

Elision

Liaison

- E. intonation

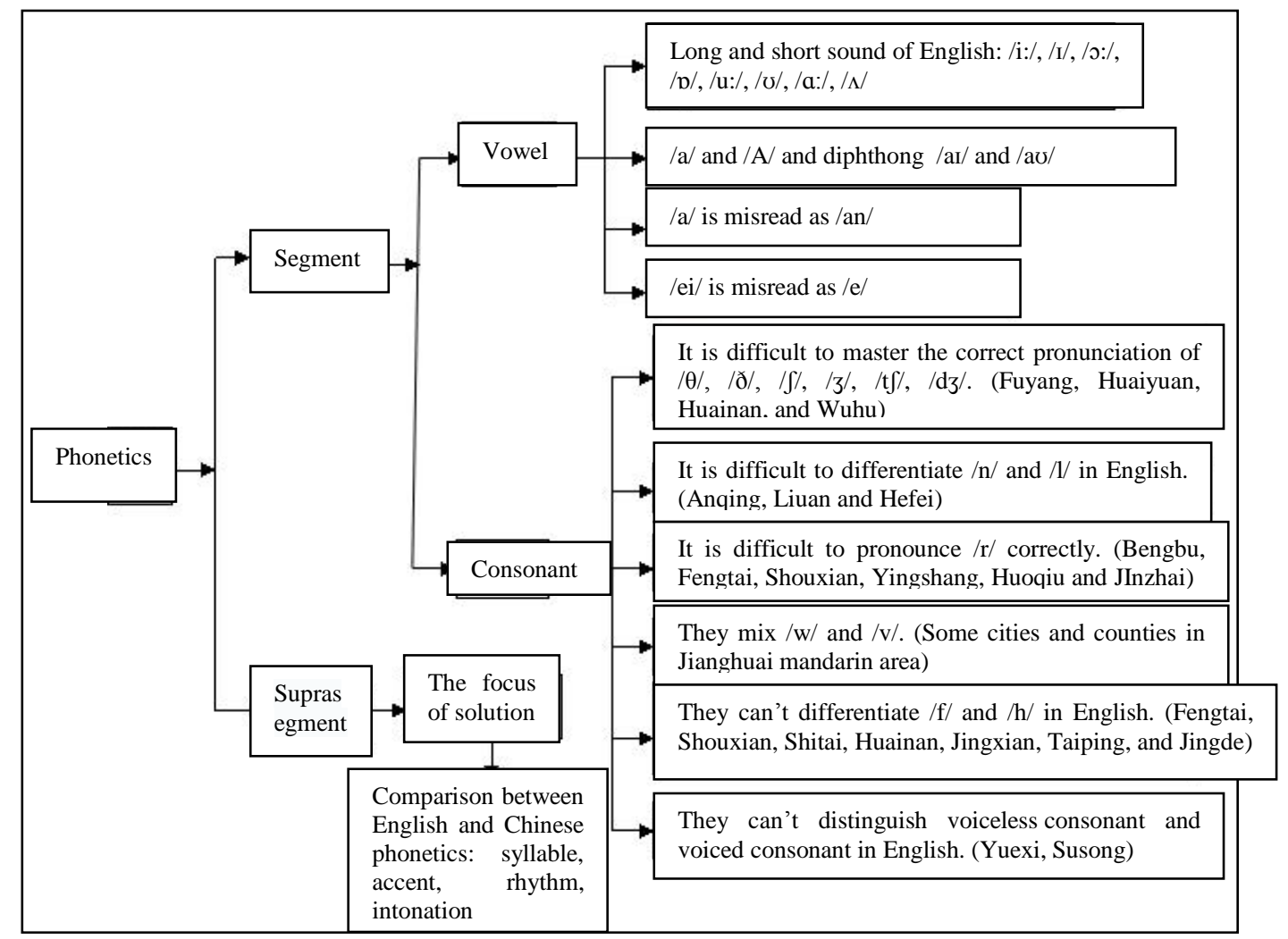

Fig. 1. Analysis of the survey data. 


\section{Probe into the Reason for Transfer Effect of Anhui Dialect on Undergraduates' English Phonetics}

The negative transfer of Chinese pronunciation is commonly found in the pronunciation of Chinese learners. Due to the regional differences, there is also a negative transfer of dialect speech to English phonetics. Students in every dialect area are deeply influenced by the dialect. In the middle school stage, they do not pay much attention to pronunciation and do not undergo systematic phonetic system training due to the purpose examination. Therefore, many students make very little practice in phonetics or adopt improper practice methods, repeatedly strengthening and consolidating erroneous phonemes. In addition, English teachers in some dialectal areas have local accents, so learners transfer the dialectal pronunciation into English pronunciation, resulting in the negative transfer of dialect pronunciation. Entering the process of phonetic learning in university, the negative transfer of dialect pronunciation negatively affects oral English, listening comprehension, English communicative competence and critical thinking skills of undergraduates. Therefore, learners are apt to have learning anxiety, and thus hate it and slack off in learning. They are reluctance to communicate with others, and the resulting impact is very prominent.

\section{THE STRATEGIES FOR PHONETIC CORRECTION}

- To Master the Basic Theory of English-Chinese Speech Synthesis Training. The essence of dialect phonetic correction, based on the standard of mandarin, comprehensively regulate and correct the deviation of dialect from the aspect of phonetics, vocabulary and grammar. Aiming at the corresponding relationship between Anhui dialect and mandarin, we should mainly focus on the phonetic theory in following aspects: relevant knowledge of four phonetic elements, physiological property of phonetics, knowledge related to syllable and phoneme, knowledge related to vowel and consonant, relevant knowledge of phonology, knowledge related to Chinese phonetic alphabet, light tone, rhotic accent, and phonetic changes knowledge.

- To Strengthen the Pronunciation Skills Training, and Repeat Practice. We should implement the theory of contrast between English and Chinese in teaching to change students' learning methods of simply imitation. We can compare the different characteristics of English and Chinese at the level of segments and syllabuses and guide students to focus on pronunciation position, the height of the tongue and the shape of the lips, transforming from simple imitation to the systematic theory study. First is to pronounce the cacuminal zh-z, ch-c, sh-s well. When pronouncing cacuminal, they need to be trained to upwarp the tongue tip against the front of the hard palate, and then the airflow bursts through the barrier formed by tongue tip and hard palate to form sound through friction. Second is to pronounce lateral [n] and [1] well. When pronouncing lateral, learners should be trained to uplift the soft palate and uvula to block the way that air flows through the nasal cavity, so that it flows out from the gap of both sides of the tongue after entering the mouth. Third is to pronounce nasal [ $\mathrm{n}]$ well by lifting the root of tongue, and leaving the soft palate drooping, so that the air flow from the nasal cavity.

- To focus on the correction training of pronunciation Cacuminal (blade-palatal) is pronounced when the tongue tip tilt up, near the back of gums or the front of hard palate, but the tongue is not obviously curve.

- To master the method of identification and memory and look for memory rule of pronunciation.

- To integrate phonetic special training and habits cultivation: whether the pronunciation, intonation, accent pronunciation are standard; whether there is obvious pronunciation defects; whether the continuous phonetic change, juncture, intonation and so on are reasonable; whether the reading is smooth and the speed of speech is appropriate.

\section{THE IMPLEMENTATION PLAN}

This paper plans to conduct through theoretical research, literature retrieval, questionnaire survey, recording, testing, data statistics and analysis and so on. Specific research ideas and methods are as follows: This study can be carried out in one university of science and engineering in Hefei by selecting 60 students from major dialect areas in Anhui Province with different majors to participate in the experiment. All of them have studied English for over 6 years. The survey identifies whether the students are from dialect area according to the birthplace of students and phonology, phoneme, and phonetics change. Samples of this survey are undergraduates who have lived in Hefei, Anqing, and Fuyang for more than 10 years, and can speak standard Mandarin and English. The college students who have regional accent and those who speak standard Mandarin are divided into the experimental group and the control group respectively. The experimental materials are 80 English words, and the tested students read sentences containing vocabularies with different vowels and live recording is made. The researcher transcribes the recording into the text material, makes statistics and conclusion to the pronunciation mark, classification, and the wrong pronunciation.

\section{CONCLUSION}

This subject intends to propose that the empirical research on the strategies of English speech correction for undergraduates in dialects in Anhui Province through theoretical research, literature search, questionnaire survey, recording, testing, data statistics and analysis is the individual cases. Through comparative analysis of English and Chinese phonetic system, combining the actual survey situation of undergraduates' pronunciation in Anhui dialect area, the existing transfer phenomenon in English phonetic learning of undergraduates in Anhui dialect area is studied and feasible suggestions for phonetic correction are put 
forward aiming at the impact of negative transfer to construct a long-term research mechanism and teaching reform project for the strategy of correcting English pronunciation in dialect area.

\section{REFERENCES}

[1] Leather, Jonathan. Second-language Pronunciation Learning and Teaching. Modern Language Quarterly [M].1983

[2] Odlin, T. Language Transfer [M]. Cambridge: Cambridge University Press, 1989

[3] Rod Ellis. Second Language Acquisition [M]. Shanghai: Shanghai Foreign Language Education Press, 2000: 30.

[4] Kager, R. Optimality Theory[M].Cambridge: Cambridge University Press, 1999.

[5] Anhui Provincial Local Records Compilation Committee. Anhui Province geography - dialect geography. Hefei: Fang zhi publishing house, 1997.

[6] Hu Zhuanglin. Linguistics tutorial. revised edition. Beijing: Peking University Press, 2001: 38.

[7] Bao Hong. Dialect pronunciation System in Anqing in Anhui Province. Journal of Anqing Teachers (social science edition), 2008 (10).

[8] Du Ruiqing, Jiang Yajun. Review of research on "English in China" in Recent Twenty Years. Foreign Language Teaching and Research, 2001 (01).

[9] He Meijia, Meng Jianghong. Study on Positive Transfer of Mother Tongue under Language share and Relevance. Journal of Shanxi Datong university (social science edition). 2012 (04)

[10] Li Huimin. Study overview of the Attribution and Characteristics of Jianghuai Mandarin. Journal of Anhui Normal University (humanities and social sciences). 2004(05).

[11] Li Xindi. Difference of Blade-alveolars and cacuminal in Dialect, Phonology and Mandarin. Journal of Chongqing Normal University (philosophy and social science edition). 2007(02).

[12] Ma Jiaying, Liu Hao, Xu Yaming. Phonetic changes and Phonetics Teaching - A Reflection on the Phonetic Teaching of English Learners. Journal of Huangshan University. 2008 (03).

[13] Ma Qiuwu. Optimality Analysis of the Structure of composite Vowels in Mandarin. Contemporary Linguistics, 2004a (2).

[14] Ma Qiuwu. The universality of surface conditioning and constraint of Optimality theory - From the Syllable fabric of Mandarin. Foreign Languages Research, 2004b (2).

[15] Shi Rujie. An Important Achievement of Huizhou Dialect Research A Review of Research on Huizhou Dialect. language research, 2000 (02).

[16] Wang Qin. "a wife" in Fuyang dialect of Anhui Province. Studies of The Chinese Language, 2010 (02).

[17] Wang Wenling, Xie Jingsi. The Constraints of Dialect on Training of Oral English capacity and entrance Cohesion Teaching. Hefei University of Technology, 2008.

[18] Zhu Yuming, Liu Feibing. The Influence of Students' Dialect Phonetics on Listening and Speaking of Foreign Language and its Countermeasures. Social Science Journal of Xiangtan University, 2002 (04).

[19] Wang Fufang, Zhang Yunqiu. Research on the Structure of "Eat + N" from the Perspective of Optimality Theory. Research in Foreign Language \& Literature, 2005(4). 